\title{
High prevalence of gastroesophageal reflux symptoms in patients with both acute and nonacute cough
}

\author{
Yoshihisa Urita \\ Toshiyasu Watanabe \\ Hiroki Ota \\ Motohide Iwata \\ Yosuke Sasaki \\ Tadashi Maeda \\ Takamasa Ishii \\ Makie Nanami \\ Asuka Nakayama \\ Hirohito Kato \\ Kazuo Hike \\ Noriko Hara \\ Masaki Sanaka \\ Yoko Nagai \\ Shuji Watanabe \\ Kazushige Nakanishi \\ Hitoshi Nakajima \\ Motonobu Sugimoto \\ Department of General Medicine \\ and Emergency Care, Toho University \\ School of Medicine, Omori Hospital, \\ Tokyo, Japan
}

\begin{abstract}
Although there have been many studies that showed a close association between gastroesophageal reflux disease (GERD) symptoms and chronic cough, it has been unknown whether acute cough is also associated with GERD. The aim of this study was to evaluate the relationship between GERD and respiratory symptoms in general practice. 1725 consecutive patients who first attended our hospital were enrolled in the present study. They were asked to respond the F-scale questionnaire regardless of their chief complaints. Over all, 656 (38\%) patients were diagnosed as GERD and 226 (13\%) had respiratory symptoms. Patients with respiratory symptoms had GERD symptoms more frequently than patients without respiratory symptoms $(\mathrm{p}<0.05)$. Forty-three $(37 \%)$ of 115 patients with acute cough and $48(43 \%)$ of 111 with nonacute cough had GERD symptoms, suggesting that development of GERD is not associated with the period of respiratory symptoms. Patients with respiratory symptoms are at a significantly increased risk of developing GERD. Whether or not treatment for GERD or respiratory diseases is useful for the prevention of respiratory symptoms and GERD, respectively, should not be driving management decisions in primary care.
\end{abstract}

Keywords: acute cough, gastroesophageal reflux, chronic cough, F-scale

\section{Introduction}

Symptoms of gastroesophageal reflux disease (GERD) are common, affecting $10 \%-30 \%$ of the population in Western countries (Holtmann 2001). Recently, the prevalence of GERD is also increasing in a Japanese population with a high prevalence of atrophic gastritis (Furukawa et al 1999). Respiratory diseases are also common causes for consultation in primary care, but the association between the two diseases remains unclear. Many previous studies of the association between the two had selection bias since data were obtained in a retrospective manner. Studies addressing the correlation between respiratory disorders and GERD symptoms in an unselected group of persons are sparse. The incidence of chronic bronchitis, asthma, and chronic pulmonary disease is 1.5 times more in patients with reflux esophagitis (el Serag and Sonnenberg 1997). Other studies demonstrated a high prevalence of GERD in asthmatic patients (Sontag et al 1990; Vaezi 2003). Thus, although there have been many studies that showed a close association between GERD symptoms and chronic cough, it has been unknown whether acute cough is also associated with GERD. The aim of this study was to evaluate the relationship between GERD and respiratory symptoms in general practice.

\section{Patients and methods}

Between September 2005 and March 2006, 1725 consecutive patients (788 men and 937 women with a mean age of 42 years) who first attended the Outpatient Department 
of General Medicine and Emergency Care of Toho University Omori Hospital were enrolled in the present study. Informed consent was obtained from all of the patients. None of the patients had a history of use of proton pump inhibitor (PPI), H2-receptor antagonist, antibiotics, steroids, or nonsteroidal antiinflammatory drugs for a period of at least two month before the investigation. Patients who had a previous history of partial gastrectomy were also excluded from the study. The study was carried out in accordance with the Declaration of Helsinki, and approved by the ethical committee at Toho University.

All of the patients who first attended our hospital were asked to respond the F-scale questionnaire regardless of their chief complaints. The questionnaire is a self-report instrument containing 12 questions written in a simple and easy-to-understand language. As reported previously by Kusano and colleagues (2004), the following definitions were used to identify symptoms in the F-scale: 1) "Do you get heartburn?"; 2) "Does your stomach feel bloated?"; 3) "Does your stomach ever feel heavy after meals?"; 4) "Do you sometimes subconsciously rub your chest with your hand?"; 5) "Do you ever feel sick after meals?"; 6) "Do you get heartburn after meals?"; 7) "Do you have an unusual sensation in your throat?"; 8) "Do you feel full while eating meals?"; 9) "Do some things get stuck when you swallow?"; 10) "Do you get bitter liquid coming up into your throat?"; 11) "Do you burp a lot?"; and 12) "Do you get heartburn if you bend over?" Symptoms frequency was measured on the following scale: never $=0$; occasionally $=1$; sometimes $=2$; often $=3$; and always $=4$. If the patient has a score of more than 7 point, GERD is considered as positive.

When a patient complains of cough that has been present for less than 3 weeks, it is considered to be an acute cough according to the ACCP evidence-based clinical practice guidelines (Braman 2006). When the cough lasts for more than 3 weeks, it is diagnosed as having nonacute cough in the present study.

All values are expressed as means \pm SD. Comparisons of groups were made using Student's $t$ test or chi-square tests as appropriate. A p value of $<0.05$ was accepted as indicating statistical significance.

\section{Results}

Over all, 656 (38\%) patients were diagnosed as GERD and 226 (13\%) had respiratory symptoms including cough, sputum, and/ or dyspnea. GERD symptoms were found in 104 of 226 patients with respiratory symptoms and in 552 of 1499 patients without respiratory symptoms. Patients with respiratory symptoms had GERD symptoms more frequently than patients without respiratory symptoms with a statistical significance $(\mathrm{p}<0.01$, Table 1). F-scale score is significantly higher in patients with respiratory symptoms $(7.3 \pm 6.2)$ than in those without respiratory symptoms $(4.4 \pm 5.8)(\mathrm{p}<0.01)$. There were no significant differences in age, male to female ratio, or the prpprtion of hypertension treated and current smokers between patients with and without respiratory symptoms (Table 1).

Among 226 patients with respiratory symptoms, 115 were diagnosed as acute cough and had a F-scale score of $6.8 \pm 6.3$. The remaining 111 patients were considered as having nonacute cough and had a F-scale score of 7.7 \pm 6.1 . There was no significant difference in F-scale score between the two groups. $43(37 \%)$ of 115 patients with acute cough and $48(43 \%)$ of 111 with nonacute cough had GERD symptoms (Table 2). The difference in prevalence of GERD between acute and nonacute cough groups did not reach statistical significance.

GERD prevalence was analyzed after excluding pharyngeal symptoms because F-scale includes a question about an unusual sensation in the throat (question 7). Comparison of GERD prevalence between patients with and without pharyngeal symptoms was shown in Table 3 . In patients with nonacute cough, those were found in $15(60 \%)$ of 25 patients with pharyngeal symptoms and in $32(38 \%)$ of 85 without them. For acute cough, $22(42 \%)$ of 53 patients with pharyngeal symptoms, and 18 (31\%) of 58 patients without pharyngeal symptoms were diagnosed as GERD. The differences in GERD prevalence between patients with and without pharyngeal symptoms did not reach a statistical significance in acute and nonacute cough groups.

\section{Discussion}

GERD refers to the abnormal exposure of the esophageal mucosa to gastric contents. Several population based-surveys

Table I Comparison of GERD prevalence and F-scale score between patients with and without respiratory symptoms

\begin{tabular}{llll}
\hline & \multicolumn{2}{l}{ Respiratory symptoms } & p-value \\
\hline No. of patient & $(+)$ & $(-)$ & \\
Age (years old) & 226 & 1499 & \\
Male:Female & $91.9 \pm 9.1$ & $43.1 \pm 8.5$ & N.S. \\
Current smoker & $79(35 \%)$ & $485(32 \%)$ & N.S. \\
Hypertension & $17(8 \%)$ & $111(7 \%)$ & N.S. \\
F-scale score & $7.3 \pm 6.2$ & $4.4 \pm 5.8$ & P $<0.01$ \\
No. of GERD & $104(46 \%)$ & $552(37 \%)$ & $\mathrm{P}<0.01$ \\
\hline
\end{tabular}

Abbreviation: GERD, gastroesophageal reflux disease. 
Table 2 Comparison of GERD prevalence and F-scale score between patients with and acute and nonacute cough

\begin{tabular}{llll}
\hline & Acute cough & Nonacute cough & p-value \\
\hline No. of patient & II5 & III & \\
F-scale score & $6.8+1-6.3$ & $7.7+/-6 . I$ & N.S. \\
No. of GERD & $43(37 \%)$ & $48(43 \%)$ & N.S. \\
\hline
\end{tabular}

Abbreviation: GERD, gastroesophageal reflux disease.

have reported typical GERD symptoms, including heartburn and acid regurgitation, at least yearly ranging from $26 \%$ to $60 \%$ (Ruth et al 1991; Staghellini et al 1999; Kennedy and Jones 2000; Diaz-Rubi et al 2004). These results are concordant with our findings although GERD symptoms in the general population are less common in the East compared to the West (Ho et al 2005; Chen et al 2005). Since Helicobacter pylori infection may result in hypochlorhydria as seen in individuals with atrophic gastritis, H. pylori-infected patients seem to be at less risk of developing GERD (Mishima et al 2005), as is generally observed in Japan with high prevalence of $H$. pylori infection. Recently, several reports showed an increasing trend of GERD in Asian countries in recent years (Ho et al 2005; Chen et al 2005; Lim et al 2005). Although it has been thought that a low acid secretion would be associated with a lower prevalence of GERD in the Far East, Kinoshita and colleagues (1997) demonstrated that gastric acid secretion in Japan is rising in both $H$. pylori-positive and - negative individuals. Actually, decreasing prevalence of $H$. pylori was observed in many countries (Banatvala et al 1993; Kosunen et al 1997; Roosendaal et al 1997) as well as in Japan (Asaka et al 1992).

On the other hand, there have been many studies that showed a close association between GERD symptoms and chronic cough (Sontag et al 1990; el Serag and Sonnenberg 1997; Vaezi 2003). Several possible mechanisms underlying a relation between GERD and respiratory symptoms have been proposed.

Two mechanisms have been postulated by which GERD may induce cough: firstly, microaspiration of gastric refluxate

Table 3The GERD prevalence in patients with and without pharyngeal symptoms in acute and nonacute cough groups

\begin{tabular}{lllll}
\hline & \multicolumn{2}{l}{ Nonacute cough } & \multicolumn{2}{l}{ Acute cough } \\
\hline $\begin{array}{l}\text { Pharyngeal } \\
\text { symptom }\end{array}$ & $(+)$ & $(-)$ & $(+)$ & $(-)$ \\
No. of patient & 25 & 85 & 53 & 58 \\
No. of GERD & $15(60 \%)$ & $32(38 \%)$ & $22(42 \%)$ & $18(31 \%)$ \\
\hline
\end{tabular}

Abbreviation: GERD, gastroesophageal reflux disease. into the lung causing an exudative mucosal reaction; and secondly, a vagally mediated distal esophageal-tracheobronchial reflex leading to bronchospasm (Ing et al 1994). The foregut and the respiratory tract have common embryological origins and share a number of reflexes. The existence of a vagally mediated esophageal-tracheobronchial reflex could explain asthma worsening after a large meal (Pellicano et al 2005). Microaspiration has also been proposed as a cause of GERD induced bronchospasm. In guinea pig model, acid perfusion of the esophagus caused neurally mediated airway inflammation (Ricciardolo et al 2004). The intimate mechanisms of acid-induced airway obstruction are dependent on activation of capsaicin-sensitive sensory nerves, with the subsequent release of tachykinins, that modulate diverse aspects of airway dysfunction (Ricciardolo et al 2004). Wilson and colleagues (1987) demonstrated direct evidence that ingestion of $200 \mathrm{~mL}$ of $0.1 \mathrm{~N} \mathrm{Hcl} \mathrm{increased} \mathrm{bronchial} \mathrm{activity.} \mathrm{Field} \mathrm{and} \mathrm{colleagues}$ (1988) reported increased minute ventilation due to sensations of discomfort during acid perfusion of the esophagus in patients with normal lung function. Thus, heightened bronchial reactivity, microaspiration, and a vagally mediated reflex mechanism are possible pathways.

The increased respiratory effort and cough augment abdominal pressure facilitating the retrograde movement of gastric contents (Field et al 2001). Changes in lung volume may alter the relationship between the diaphragm and lower esophageal sphincter (LES) disrupting the physiological activity (Stein et al 1980). The effect of asthma medications as well as the negative intrathoracic pressure produces during the asthma attacks may overcome the protective effect of the LES, resulting in increased GERD (Vaezi 2003). Theophylline, a bronchodilator, has been shown to stimulate gastric acid secretion and lower the LES pressure (Harding 2001). Exposure to small amounts of acid has been proposed to result in impaired laryngopharyngeal sensitivity and thereby potentially increasing the risk of aspiration (Harding 2001). Using prolonged esophageal $\mathrm{pH}$ monitoring, chronic persistent cough without obvious pulmonary etiology was likely to be associated with episodes of otherwise asymptomatic gastroesophageal reflux (Phua et al 2005). Likewise, cough from any cause can precipitate gastroesophageal reflux (Ing et al 1991). From these viewpoints, the present study on relationship between GERD symptoms and acute cough was proposed.

Similarly to the results of previous studies (Sontag et al 1990; el Serag and Sonnenberg 1997; Vaezi 2003), patients with respiratory symptoms had GERD symptoms more frequently than patients without respiratory symptoms with a statistical significance in the present study. Although the 
GERD prevalence is higher in patients with nonacute cough than in those with acute cough, there was no significant difference. This suggests that even acute cough can cause an increase in intra-abdominal pressure which in turn may promote movement of gastric contents into the esophagus. It seems unlikely that GERD might cause acute cough because GERD is a chronic disease. Cough induced by GERD should be prolonged. Avidan and colleagues (2001) investigated the temporal association between coughing or wheezing and reflux episodes in asthmatics and concluded that even though occasional coughing can lead to reflux, the opposite is far more common. Some patients sometimes complain of a persistent cough following symptoms of an upper respiratory tract infection in clinical practice. When cough has been present for at least 3 weeks, but not more than 8 weeks, a diagnosis of postinfectious cough should be considered (Braman 2006). The frequency of postinfectious cough increases to $25 \%$ to $50 \%$ (Davis et al 1995) and gastroesophageal reflux is considered to be one of its mechanisms, suggesting that medical treatment of acid reflux in acute phase of cough may reduce postinfectious cough although the pathogenesis is often multifactorial.

An epidemiological study on GERD could be considered accurate only when the whole study population undergoes invasive procedure. However, this would dramatically reduce the number of participants and prejudice the response rate of the study. F-scale, therefore, seems to be the most appropriate test for general practice patients in the management of GERD because it has made no use of endoscopy. F-scale covers various symptoms related to functional dyspepsia, as well as GERD. Among 12 questions in F-scale, the question 7 is closely related to sore throat, which is one of the most common symptoms observed in patients with an upper respiratory tract infection. It is likely that GERD prevalence in patients with acute cough is influenced by the presence of sore throat. We therefore analyzed the GERD prevalence after excluding the question 7, and there were no differences in GERD prevalence between patients with and without pharyngeal symptoms in acute and nonacute cough groups. This indicates that sore throat does not affect the results of this study.

In conclusion, patients with respiratory symptoms are at a significantly increased risk of developing GERD. There was no difference in GERD prevalence between patients with acute and nonacute cough, suggesting that development of GERD is associated with the presence of respiratory diseases regardless of the period of respiratory symptoms. It is more important that the association between GERD and acute cough be more widely recognized in the primary care physicians.

\section{Disclosure}

The authors report no conflicts of interest in this work.

\section{References}

Asaka M, Kimura T, Kudo M, et al. 1992. Relationship of Helicobacter pylori to serum pepsinogens in an asymptomatic Japanese population. Gastroenterology, 102:760-6.

Avidan B, Sinnenberg A, Schnell TG, et al. 2001. Temporal associations between coughing or wheezing and acid reflux in asthmatics. Gut, 49:767-72.

Banatvala N, Mayo K, Megraud F, et al. 1993. The cohort effect and Helicobacter pylori. J Infect Dis, 168:219-21.

Braman SS. 2006. Postinfectious cough, ACCP evidence-based clinical practice guidelines. Chest, 129:S138-46.

Chen M, Xiong L, Chen H, et al. 2005. Prevalence, risk factors and impact of gastroesophageal reflux disease symptoms: A population-based study in South China. Scand J Gastroenterol, 40:759-67.

Davis SF, Sutter RW, Strebel PM, et al. 1995. Concurrent outbreaks of pertussis and Mycoplasma pneumoniae infection: clinical and epidemiological characteristics of illnesses manifested by cough. Clin Infect Dis, 20:621-8.

Diaz-Rubio M, Moreno-Elola-Olaso C, Rey E, et al. 2004. Symptoms of gastro esophageal reflux; prevalence, severity, duration and associated factors in a Spanish population. Aliment Pharmacol Ther, 19:95-105.

el Serag HB, Sonnenberg A. 1997. Comorbid occurrence of laryngeal or pulmonary disease with esophagitis in United States military veterans. Gastroenterology, 113:55-760.

Field SK, Evans JA, Price LM. 1988. The effects of acid perfusion of the esophagus on ventilation and respiratory sensation. Am J Respir Crit Care Med, 157(4 Pt 1):1058-62.

Field SK, Field TS, Cowie RL. 2001. Extraesophageal manifestations of gastroesophageal reflux. Minerva Gastroenterol Dietol, 47:137-50.

Furukawa N, Iwakiri R, Koyama T, et al. 1999. Proportion of reflux esophagitis in 6010 Japanese adults-prospective evaluation by endoscopy. J Gastroenterol, 34:441-4.

Harding SM. 2001. Gastroesophageal reflux, asthma, and mechanisms of interaction. Am J Med, 111(Suppl):S8-S11.

Ho KY, Chan YH, Kang JY. 2005. Increasing trend of reflux esophagitis and decreasing trend of Helicobacter pylori infection in patients from a multiethnic Asian country. Am J Gastroenterol, 100:1923-8.

Holtmann G. 2001. Reflux disease: the disorder of the third millennium. Eur J Gastroenterol Hepatol, 13(Suppl 1):S5-11.

Ing AJ, Ngu MC, Breslin AB. 1991. Chronic persistent cough and gastroesophageal reflux. Thorax, 46:479-83.

Ing AJ, Ngu MC, Breslin AB. 1994. Pathogenesis of chronic persistent cough associated with gastroesophageal reflux. Am J Respir Crit Care Med, 149:160-7.

Kennedy T, Jones R. 2000. The prevalence of gastro-esophageal reflux symptoms in a UK population and the consultation behavior of patients with these symptoms. Aliment Pharmacol Ther, 14:1589-94.

Kinoshita Y, Kawanami C, Kishi K, et al. 1997. Helicobacter pylori independent chronological change in gastric acid secretion in the Japanese. Gut, 41:452-8.

Kosunen TU, Aromaa A, Knekt P, et al. 1997. Helicobacter antibodies in 1973 and 1994 in the adult population of Vammala, Finland. Epidemiol Infect, 119:29-34.

Kusano M, Shimoyama Y, Sugimoto S, et al. 2004. Development and evaluation of FSSG: frequency scale for the symptoms of GERD. J Gastroenterol, 39:888-91.

Lim SL, Goh WT, Lee JM, et al; Community Medical GI study Group. 2005. Changing prevalence of gastroesophageal reflux with changing time: Longitudinal study in an Asian population. J Gastroenterol Hepatol, 20:995-1001.

Mishima I, Adachi K, Arima N, et al. 2005. Prevalence of endoscopically negative and positive gastroesophageal reflux disease in the Japanese. Scand J Gastroenterol, 40:1005-9. 
Pellicano R, Ponzetto A, Smedile A, et al. 2005. Gastro-esophageal reflux disease and asthma: would be possible to improve therapy on the basis of what is now known? Panminerva Med, 46:135-40.

Phua SY, McGarvey LP, Ngu MC. 2005. Patients with gastroesophageal reflux disease and cough have impaired laryngopharyngeal mechanosensitivity. Thorax, 60:488-91.

Ricciardolo FL, Gaston B, Hunt J. 2004. Acid stress in the pathology of asthma. J Allergy Clin Immunol, 113:610-19.

Roosendaal R, Kuipers EJ, Buitenwerf J, et al. 1997. Helicobacter pylori and the birth cohort effect: evidence of a continuous decrease of infection rates in childhood. Am J Gastroenterol, 92:1480-2.

Ruth M, Mansson I, Sandberg N. 1991. The prevalence of symptoms suggestive of esophageal disorders. Scand J Gastroenterol, 26:73-81.
Sontag SJ, O’Connell S, Khandelwal S, et al. 1990. Most asthmatics have gastroesophageal reflux with or without bronchodilator therapy. Gastroenterology, 99:613-20.

Stanghellini V. 1999. Three-month prevalence rates of gastrointestinal symptoms and the influence of demographic factors: results from the Domestic/International Gastroenterology Surveillance Study (DIGEST). Scand J Gastroenterol, 231(Suppl):20-8.

Stein MR, Towner TG, Weber RW, et al. 1980. The effect of theophylline on the lower esophageal sphincter pressure. Ann Allergy, 45:238-41.

Vaezi MF. 2003. Extraesophageal manifestations of gastroesophageal reflux disease. Clin Cornerstone, 5:32-8.

Wilson NM, Chudry N, Silverman M. 1987. Role of the esophagus in asthma induced by the ingestion of ice and acid. Thorax, 42:506-10. 
